\title{
Cooperative Target Detection in a Network of Single-Channel Radar Sensors
}

\author{
Maximilian Steiner, Karim S. Osman, and Christian Waldschmidt
}




\title{
Cooperative Target Detection in a Network of Single-Channel Radar Sensors
}

\author{
Maximilian Steiner, Karim S. Osman, and Christian Waldschmidt \\ Institute of Microwave Engineering, Ulm University, 89081 Ulm, Germany \\ Email: Maximilian.Steiner@uni-ulm.de
}

\begin{abstract}
In automotive sensor networks the spatial distribution of the radar sensors allows to unveil additional information about the target objects. For example, the different angles to a target provide an increased robustness, and the wide sensor distances allow for a single snapshot motion estimation. This is even possible with single-channel radar sensors, but the reliability of the output highly depends on the condition that single scattering points are jointly detected. This paper presents an algorithm which associates single detections of a target over multiple sensors by utilizing the ego-motion of the vehicle. Further, it is shown how all target detections can be assessed and how reliable detections can be identified to improve subsequent processing steps like target localization. The proposed processing is validated by radar measurements at $77 \mathrm{GHz}$.
\end{abstract}

\section{INTRODUCTION}

Over the last years radar sensors became essential components of many driver assistance systems. Multiple radar sensors around a car are already used for dedicated assistance systems without being jointly evaluated. These trends towards a $360^{\circ}$ coverage of the traffic scene around the car make sensor networks very interesting. This work covers a network of single-channel radar sensors. Such simple sensors allow to estimate the range and radial velocity of a target. In contrast to state-of-the-art automotive radars with multiple receive channels, the angle information of a target has to be calculated jointly from the measurements of multiple sensors observing a common field of view (FoV). A presumption for the related localization algorithms, like the multilateration, is that all sensors detect a common scattering point [1]. However, this is hardly met in automotive scenarios with extended targets [2]. Here, the probability of joint detections is lowered due to the comparably high range resolution and the possibly large spatial distribution of the sensors, as well as the angle dependent radar cross-sections of targets [3]. This paper shows an approach to associate the detections of stationary targets between the sensors that observe a common FoV. Further, a robustness measure for each target is derived, which allows to differentiate between point targets and extended targets.

\section{System SetuP}

The proposed system utilizes very simple single-channel radar sensors. These sensors are operated with a chirp sequence modulation [4] that allows to estimate the range and radial velocity of a target.

Multiple simple sensors are spatially distributed on a moving platform, e.g. a car, as depicted in Fig. 1. At

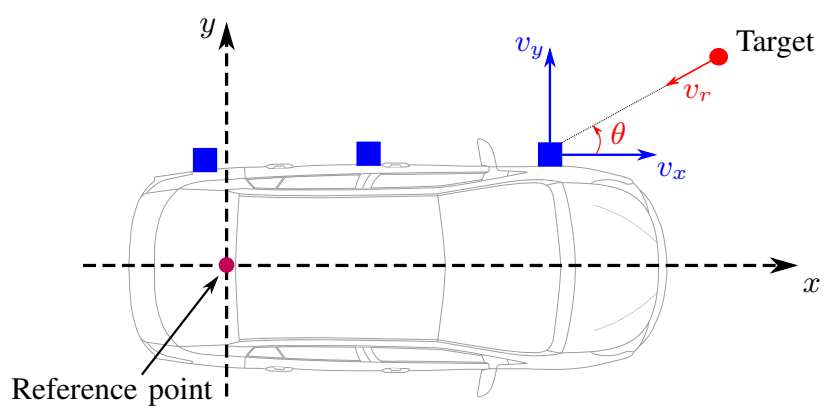

Fig. 1. Exemplary spatial distribution of 3 sensors around a car [5].

least 3 sensors cover a common $\mathrm{FoV}$ to enable the use of multilateration techniques. A joint trigger signal ensures simultaneous measurements of all sensors in the network. The center frequencies of all sensors are shifted respectively to avoid interference among sensors in the network. Hence, it can be assumed that all sensors provide independent monostatic measurements of a common scene.

\section{Cooperative TARget Detection}

The flowchart of the cooperative target detection algorithm is briefly illustrated in Fig. 2. In the following, each step is described in detail.

\section{A. Target Extraction}

The input of the algorithm is the raw time-domain data of each sensor. This data has a 2D-shape with the size of (samples $\times$ chirps). In the first processing step, the 2D discrete Fourier transform is used to derive the range and radial velocity information, resulting in a range-Doppler matrix for each sensor [4].

A 2D-ordered statistic constant false alarm rate (OS CFAR) [6] is used in the range-Doppler domain to extract the targets detected by each sensor. Several detections are usually extracted per target after applying a CFAR algorithm. Thus, a $2 \mathrm{D}$ peak search ensures the extraction of nearby targets. A low decision threshold is applied to the sensors while extracting the targets to gather as many targets as possible. This is favorable when estimating the ego-motion, as well as helping to provide a better knowledge about the surrounding targets in the scene.

\section{B. Ego-motion Estimation}

The next step is to estimate the ego-motion of the vehicle, whereby the approach in [7] is followed. The range information of the detected targets is used by the multilateration technique 




Fig. 2. Algorithm overview. The dashed processing step is individual to every sensor, whereas the following steps include all sensors jointly.

to calculate the intersection points between the range circles. This results in true target and ghost target intersections [8]. The angle between each intersection point and the corresponding sensors is calculated. Ghost targets arising from inter-range intersections lead to incorrect angles, thus, an increasing number of ghost targets would consequently result in an inaccurate ego-motion estimation. For that reason, a spatial filtering technique for point targets, referred to as matched filter, is first used to reduce the ghost intersections before applying the random sample consensus (RANSAC) algorithm as explained in [7]. More reliable results were achieved after applying this filter.

After estimating the sensors velocity profile, it is now possible to calculate the angle at which a stationary target is located using the detection of a single sensor only. This is done using the sensor velocity vector along with the radial velocity of a target relative to the sensor. Using the measured range and calculated angle, the target locations can also be computed.

\section{Target Association}

After determining the location of the targets detected by each of the sensors, the aim is to extract a common target list out of the individual target lists. This process involves several steps that are explained next.

\section{1) Partners Computation}

To find out if a target is common between all sensors, one of the sensors $S_{i}$ is set as the master sensor while its detection $T_{S_{i}}$ is assumed to be a true target. The position of $T_{S_{i}}$ relative to the other sensors $S_{j},(j \neq i)$, is then computed. They are referred to as the "partners" of $T_{S_{i}}$. Partners can be seen as the supposed target locations if all sensors are detecting the same target. Partners of a target are computed using the calculated target locations, the known sensor positions as well as the estimated ego-motion. This procedure is repeated such that each of the sensors in the network is once set as the master sensor, whereby its detected target is assumed to be the true target and the partners of this target are computed accordingly.

\section{2) Target Association}

By comparing the detected targets and the computed partners of each sensor, targets detected by multiple sensors can be associated as illustrated in Fig. 3. Deviations between the detections and partners of a sensor usually occur due to inaccuracies in the estimated ego-motion, target geometry as well as the limited radar resolution. Thus, some tolerances should be allowed when comparing the detections and partners of a sensor. This comparison is performed in the range-Doppler domain. An error function is used to combine the deviations in the range and Doppler dimensions, in which the deviations are weighted based on their respective radar resolutions. This weighted error function is normalized to the maximum tolerable range and Doppler deviations and is expressed as

$$
w_{n_{j i}}\left(\epsilon_{R}, \epsilon_{v_{r}}\right)=\frac{\left(\frac{\epsilon_{R}}{\Delta R}+\frac{\epsilon_{v_{r}}}{\Delta v_{r}}\right)}{\left(\frac{\mu_{R}}{\Delta R}+\frac{\mu_{v_{r}}}{\Delta v_{r}}\right)} .
$$

Here, $\epsilon_{R}$ and $\epsilon_{v_{r}}$ are the deviations in range and radial velocity between the detection $T_{S_{j}}$ of sensor $S_{j}$ and the partner calculated from the true target $T_{S_{i}}$ relative to $S_{j}$ respectively. The parameters $\Delta R$ and $\Delta v_{r}$ represent the respective resolutions of a chirp sequence radar. Given that the deviation between a target and its corresponding partner relative to a sensor is smaller than the allowed range and Doppler tolerance $\left(\mu_{R}, \mu_{v_{r}}\right)$, targets detected by different sensors can be cooperatively related to extract a common target list.
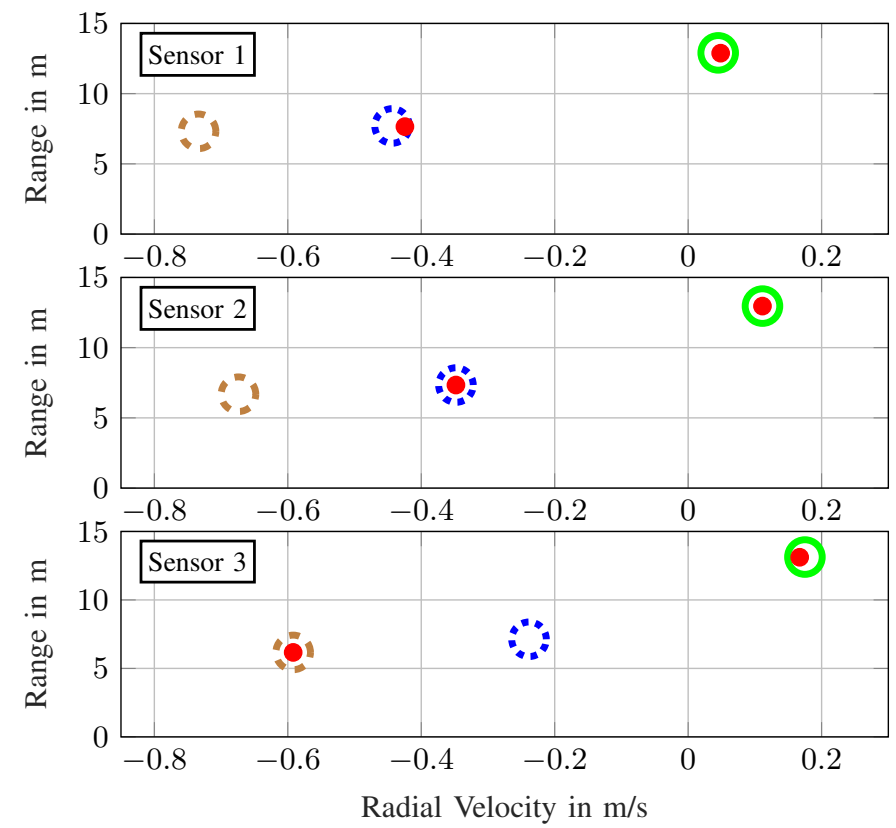

Fig. 3. The range-velocity plots of the detected targets $(0)$ and the computed partners (O) relative to each sensor. $(\mathbf{O}),(\vdots)$ and $(*)$ correspond to a triple, double, and single detected targets respectively.

\section{3) Master Sensor Assignment}

After extracting a common list of targets, one master sensor needs to be assigned to each target in the scene. This is done using the normalized weighted error function in (1). The sensor leading to the minimum sum of errors between the targets and partners is assigned as the master sensor.

Considering sensor $S_{i}$ as the master sensor, the sum of the normalized weighted errors between the detections $T_{S_{j}}$ and 
the estimated partners of $T_{S_{i}}$ relative to the sensors $S_{j}$ is first calculated. The minimum sum of errors, when each of the sensors is set as the master sensor, can be calculated as

$$
w_{n, \min }\left(\epsilon_{R}, \epsilon_{v_{r}}\right)=\min _{\forall i \in N_{s}}\left(\sum_{j=1}^{N_{s}} w_{n_{j i}}\left(\epsilon_{R}, \epsilon_{v_{r}}\right)\right) \text {. }
$$

Here, $N_{s}$ is the number of sensors and the sensor $S_{i}$ corresponding to $w_{n, \text { min }}$ is assigned as the master sensor for the common target.

An example of the association procedure is illustrated in Fig. 3, in which only that case is depicted where each target is already assigned to a master sensor. As it can be observed, not all targets are detected by all sensors. Hence, common targets are divided into triple, double, and single detected targets based on the number of detecting sensors.

\section{Clutter Suppression}

The low decision threshold applied while extracting the targets detected by the sensors leads to an increase in the probability of false alarms. To counter that, a simple clutter suppression technique is applied to reduce the detections that might correspond to clutter.

Targets detected by only one sensor are the least reliable among all targets detected by the sensors. Therefore, clutter suppression based on a histogram approach is applied to all single detections. A histogram is computed based on the signal-to-noise ratio (SNR) to study the distribution of the targets. From that, the upper bound of the most frequent SNR bin is set as a threshold to minimize the single detections. Any target that is detected by a single sensor with an SNR less than the assigned threshold is removed from the common target list and is no more considered in the further processing. This significantly reduces the number of single detected targets which minimizes the clutter detection probability.

\section{E. Target Localization}

The range information of the detections corresponding to a target are combined using the multilateration technique to determine the unique target locations. The location of a triple detected target is optimized in the region of intersection of the three range circles such that the distance between the target location and all range circles is minimized. For a double detected target, the location is simply considered as the intersection between the corresponding range circles that lies within the FoV of the sensors.

\section{F. Target Assessment}

An assessment is performed to evaluate how robust the detections of the sensors are. This provides a very useful information to the higher level processing algorithms about the surrounding targets in the traffic scene. The assessment is based on four scores that are dependent on different criteria. The values of the scores are defined in the range of $[0,1]$ and are all combined to calculate the robustness score.

Detection score: A target is ideally detected by all sensors which cover this angle area. Hence, the more sensors detecting a certain target, the higher is its detection score.
Inlier score: Detections that were used to estimate the ego-motion have a combination of range and radial velocity that supports the common motion profile [7]. Targets that are associated with such detections have a higher reliability.

Weight score: The deviations between the detected targets and partners can also be used as a score to assess the robustness. Thus, the minimum sum of the weighted error in (2) is used to calculate the weight score of a target. The minimum sum of errors is averaged over the number of detecting sensors for a fair comparison between the targets.

SNR score: The SNR score of a target is dependent on the SNR of the received signal after reflection. This in turn depends on the type of the reflecting target, its radar cross-section (RCS) and the angle of arrival of the received signal. The SNR score of a target is calculated as the maximum SNR of the corresponding detections normalized to the maximum SNR of all targets.

Robustness score: The overall score is calculated as the sum of all scores whereby the detection, inlier, and weight scores are assigned with their full weight, while the SNR score is assigned with half of its weight only. This is due to the general fluctuating and unstable behavior of the SNR. The robustness score is normalized to the maximum sum of all scores, which is 3.5 .

\section{Measurement Results}

The proposed cooperative target detection algorithm is tested on real measurement data. The location of all targets detected by the sensors in one of the measurement frames is illustrated in Fig. 4 against the ground-truth locations. Three $77 \mathrm{GHz}$ sensors are mounted on a platform, that was moved along the $x$-axis. The target scenario contained 5 corner reflectors and a stationary car. It can be observed that 4 of the corner reflectors were detected by the sensors, while one of the reflectors was not detected due to the blockage of the parked car to the line of sight (LOS). Furthermore, 5 points corresponding to the car were detected. By examining the preceding and subsequent frames, it can be deduced that detections not corresponding to ground-truth targets are not stable and could correspond to clutter from the street or from an asphalt-grass edge that was in the scene.

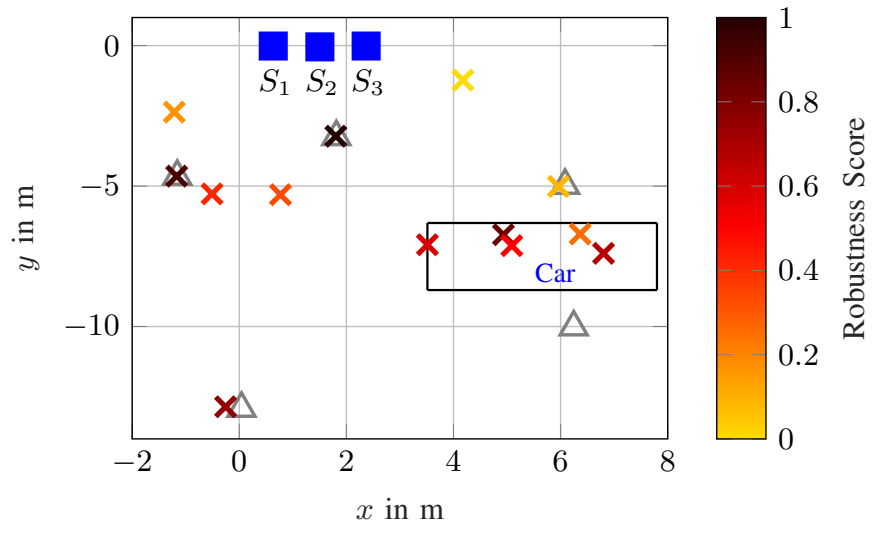

Fig. 4. Illustration of the estimated $(\mathbf{X})$ vs. ground-truth target locations. Point targets $(\triangle)$ and extended targets (Car) are depicted separately. 


\section{A. Ego-motion Estimation}

The accurate estimation of the velocity profile is crucial for the cooperative target detection. By calculating the error between the estimated ego-motion and the ground-truth motion measured by an accurate GNSS and IMU, it can be observed from the results illustrated in Fig. 5 that an estimation error less than $0.39 \mathrm{~m} / \mathrm{s}$ is achieved in $95 \%$ of the frames. This shows that the applied decision threshold enables the extraction of reliable detections which in turn allows for a very accurate snapshot motion estimation in majority of the frames.

A big advantage of the proposed assessment method is that even the failure in estimating the ego-motion can still be identified from the detection score of the targets. This is because an inaccurate ego-motion estimation would lead to the wrong association of the detections between the sensors. A failure in the cooperative relation of the sensors targets will in turn cause the number of single detections to increase. Such an effect could be directly observed in the mean of the detection score of the targets (cf. Section III-F), which is significantly reduced by the increase in the estimation error as illustrated in Fig. 5.



Fig. 5. The estimation error of the ego-motion vs. mean detection score (MDS) of the detected targets per frame.

\section{B. Assessment Scores}

Another advantage of the presented assessment method is that it allows to differentiate between single-point and extended targets. This is possible due to the different properties of both target groups which are utilized by the assessment scores. The ego-motion is based on the assumption that all sensors are detecting the same exact point of a target. This affects the detection, inlier, and weight scores of a target. The SNR scores of point targets are also higher than that of the extended ones due to the RCS of the objects.

The results illustrated in Fig. 6 show that there is a significant difference between the mean of the robustness score of the point targets to that of the extended ones. The value of the robustness scores of the point targets is $81.9 \%$ on average, while that of the extended targets is $34.5 \%$. In other words, the difference in the mean of the robustness scores of both target groups is $47.4 \%$ on average. Furthermore, it could be observed from Fig. 6, that there exists a separation distance of approximately $8.7 \%$ between the minimum score of the point targets and the maximum of the extended ones. Such a large

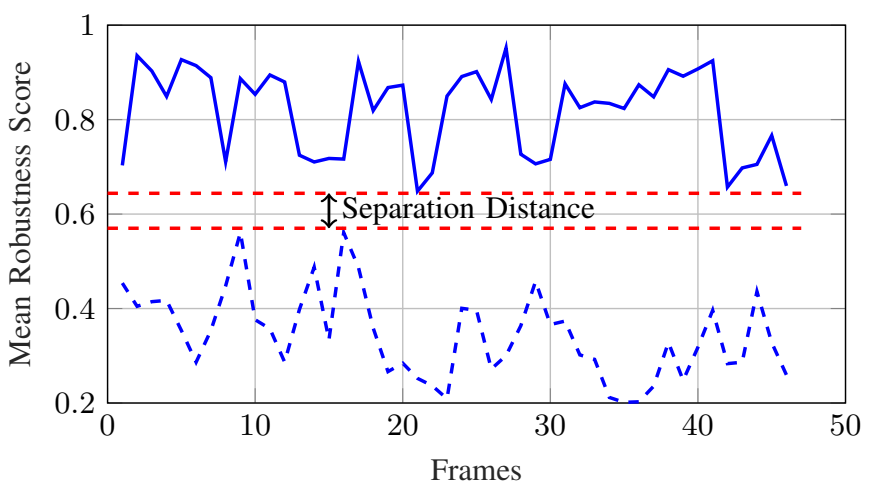

Fig. 6. The mean robustness score of point targets (-) vs. extended targets (- - ). The minimum and maximum scores of the target groups are separated.

difference in the overall assessment score could be used to classify the targets into single-point and extended targets, a feature that could be used by the higher level algorithms to process each of the target groups accordingly.

\section{CONCLUSION}

An approach for the association of stationary target detections between different radars in a network of very simple sensors is shown. It utilizes the ego-motion of the vehicle and does not depend on localization errors of the multilateration. Single snapshot measurements are sufficient and no tracking is needed for the proposed approach. The presented assessment method allows to directly distinguish between stationary extended and point targets. This information is valuable for subsequent algorithms, like target localization or target classification. The approach was evaluated with radar measurements at $77 \mathrm{GHz}$.

\section{ACKNOWLEDGMENT}

The authors thank Rik Bähnemann, Autonomous Systems Lab ETH Zürich for the support with the ground-truth measurements.

\section{REFERENCES}

[1] M. Mirbach and W. Menzel, "A simple surface estimation algorithm for UWB pulse radars based on trilateration," in IEEE International Conference on Ultra-Wideband (ICUWB), Sep. 2011, pp. 273-277.

[2] A. Frischen, J. Hasch, and C. Waldschmidt, "Contour recognition with a cooperative distributed radar sensor network," in IEEE MTT-S International Conference on Microwaves for Intelligent Mobility (ICMIM), Apr. 2015, pp. 1-4.

[3] I. Matsunami, R. Nakamura, and A. Kajiwara, "RCS measurements for vehicles and pedestrian at 26 and 79GHz," in 6th International Conference on Signal Processing and Communication Systems (ICSPCS), Dec. 2012, pp. $1-4$.

[4] A. G. Stove, "Linear FMCW radar techniques," IEE Proceedings F Radar and Signal Processing, vol. 139, no. 5, pp. 343-350, Oct. 1992.

[5] Vecteezy. (2018) Free Illustration of a Hybrid Car. [Online]. Available: www.vecteezy.com/vector-art/113748-free-illustration-of-hybrid-car

[6] H. Rohling, "Radar CFAR Thresholding in Clutter and Multiple Target Situations," vol. AES-19, no. 4, Jul. 1983, pp. 608-621.

[7] M. Steiner, O. Hammouda, and C. Waldschmidt, "Ego-Motion Estimation using Distributed Single-Channel Radar Sensors," in IEEE MTT-S International Conference on Microwaves for Intelligent Mobility (ICMIM), Apr. 2018, pp. 1-4.

[8] D. Oprisan and H. Rohling, "Tracking systems for automotive radar networks," in RADAR, Oct. 2002, pp. 339-343. 